\title{
Reference Equations for the Six-Minute Walk Distance in Healthy Korean Adults, Aged 22-59 Years
}

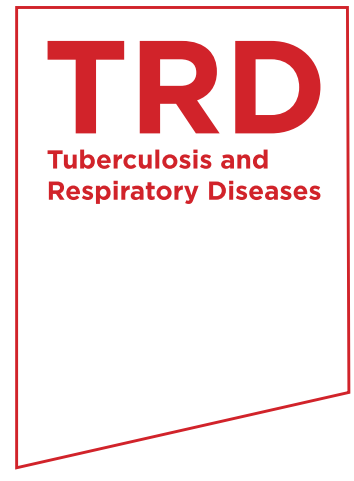

\author{
Ah Lim Kim, M.D., Jae Choon Kwon, M.D., In Park, M.D., Ji Na Kim, M.D., Jong Min Kim, M.D., Bi \\ Na Jeong, M.D., Sung Ken Yu, M.D., Byung Ki Lee, M.D., Ph.D. and Yeon Jae Kim, M.D., Ph.D. \\ Department of Internal Medicine, Daegu Fatima Hospital, Daegu, Korea
}

Background: The six-minute walk test has been widely used in people with chronic cardiopulmonary disorders as an outcome assessment with regards to therapeutic or prognostic determinants. This study was undertaken to determine the six-minute walk distance $(6 \mathrm{MWD})$ in a sample of healthy Koreans and to create a reference equation. We also compared the 6MWD of our cohort with previously published equations.

Methods: Two hundred fifty-nine healthy subjects (95 males) aged 22-59 years performed two walking tests using a standardized protocol. 6MWD was defined as the greatest distance achieved from the two tests. The effect of anthropometrics on the 6MWD was also investigated.

Results: The average 6MWD was $598.5 \pm 57.92 \mathrm{~m}$, with significantly longer distances by males $(628.9 \pm 59.51 \mathrm{~m})$ than females $(580.9 \pm 47.80 \mathrm{~m})(\mathrm{p}<0.001)$. Age, height, weight, and body mass index were significantly correlated with 6MWD in univariate analysis. Stepwise multiple regression showed height to be single independent predictor of $6 \mathrm{MWD}\left(\mathrm{r}^{2}=0.205\right.$, $\mathrm{p}<0.001)$. The reference equations derived in Caucasian and North African populations tend to overestimate the distance walked by Korean subjects, while Asian equations underestimate it.

Conclusion: The average 6MWD in these Korean populations was $600 \mathrm{~m}$. The regression equation revealed that individual's height was the most significant predictor of distance, explaining $20.5 \%$ of the distance variance.

Keywords: Exercise Tolerance; Exercise Test; Physical Endurance; Reference Values; Healthy Volunteers

\section{Introduction}

The measurement of exercise capacity is frequently used

Address for correspondence: Yeon Jae Kim, M.D., Ph.D.

Department of Internal Medicine, Daegu Fatima Hospital, 183 Ayang-ro, Dong-gu, Daegu, 701-600, Korea

Phone: 82-53-940-7462, Fax: 82-53-954-7417

E-mail: kimyeonjae@gmail.com

Received: Jan. 12, 2014

Revised: Mar. 20, 2014

Accepted: Apr. 7, 2014

(c) It is identical to the Creative Commons Attribution Non-Commercial License (http://creativecommons.org/licenses/by-nc/3.0/).

Copyright (c) 2014

The Korean Academy of Tuberculosis and Respiratory Diseases.

All rights reserved. to assist clinicians in evaluating functional capacity, assessing prognosis, and evaluating response to treatment in patients with various cardiopulmonary diseases ${ }^{1-3}$. Maximum oxygen consumption obtained via incremental laboratory tests performed in a cycle ergometer or treadmill is more useful in assessing exercise capacity ${ }^{4-6}$; however, it is not easily available because of the high cost of equipment and the complexity of test procedures.

The six-minute walk test (6MWT) is a simpler and less expensive tool, and patients are more willing to tolerate it as a method of assessing the cardiorespiratory fitness ${ }^{7}$. It provides a valid assessment of functional performance in persons with chronic cardiopulmonary disorders and has been broadly used in clinical settings. A variety of reference equations predicting six-minute walk distance (6MWD) in healthy subjects has been reported ${ }^{8-12}$, but most studies have been undertaken in white populations. These equations may not be suitable for 
predicting 6MWD in Asian populations, because the equations overestimate the distance ${ }^{13,14}$. Although some clinical studies using 6MWD as an outcome assessment have been reported in this country ${ }^{15-18}$, so far no study exists looking at 6MWD reference equations for healthy subjects.

The aims of present study were the following: 1) to determine 6MWD in a healthy sample of Koreans aged between 22 and 59 years, 2) to identify demographic and anthropometric factors contributing to 6MWD and establish a reference equation, and 3) to examine the differences between walking distances observed in our cohort and previously published equations.

\section{Materials and Methods}

\section{Healthy subjects}

Two hundred fifty-nine healthy Korean volunteers between the ages of 22 and 59 years were randomly recruited to participate in this descriptive, prospective, cross-sectional study. Subjects were medical students, hospital workers, visitors, and employees of a local private company. The study was conducted according to the Declaration of Helsinki and Good Clinical Practice guidelines. Written, informed consent was obtained from each healthy subject before participation in the study, and the study protocol was approved by local hospital institutional review board (approval number: DFH090T057). Data from each subject included age, sex, height, weight, smoking history, medication use, medical history, physical examination, and spirometry. Any of the following is regarded as a criterion for exclusion from the study: medical histories that were indicative of metabolic, cardiovascular, or pulmonary disease, current or ex-smokers with a smoking history of at least 10 pack-years, upper respiratory tract infections within the last 4 weeks, conditions that could affect walking, resting blood pressures higher than 140/90 $\mathrm{mm} \mathrm{Hg}$, heart rates higher than 100 beats per minute, body mass index (BMI) greater than 35 , or evidence of ventilatory impairment as measured by spirometry (i.e., forced expiratory volume in one second $\left(\mathrm{FEV}_{1}\right)$ /forced vital capacity (FVC) values lower than $70 \%$, of normal predicted value).

\section{Six-minute walk tests}

Two six-minute walks were performed for all healthy subjects on the same day, with 30-minute rests in between walks. The 6MWT protocol used was based on published guidelines $^{7}$, and $30 \mathrm{~m}$-long hospital corridor with marks at $3 \mathrm{~m}$ intervals was used. Each 6MWT was supervised by a medical doctor, who did not walk alongside the healthy subject. A standard explanation was provided just before the first 6MWT: "Walk as quickly as you can along the corridor for 6 minutes to cover as much ground as possible. You may stop if you have to, but continue again as soon as you are able. During the test, oxygen saturation and heart rate were measured by wearable transcutaneous pulse oximetry (Nonin WristOx 3100; Nonin, Plymouth, MN, USA). Subjects were allowed to stop if they developed symptoms of dyspnea, chest pain, leg cramps, or dizziness, but were encouraged to continue walking as soon as they could. At the ends of each minute subjects were given feedback on the elapsed time and standardized encouragement in the form of statements such as "you're doing well," and "do your best." Before and after each 6MWT, pulse rate, blood pressure, oxygen saturation, and Borg value were recorded. Subjects were asked to indicate their level of dyspnea perception using a modified 12-point Borg scale, wherein each score (i.e., ranging from 0 to 10) is illustrated by a printed figure. The maximum predicted heart rate $(\mathrm{mHR})$ was calculated as 220 minus age.

\section{Spirometry}

Height and weight were measured using a calibrated scale, and BMI for each subject was calculated before spirometry. Subjects were asked to perform at least three measures, and the largest value was recorded for analysis as recommended by the American Thoracic Society guidelines ${ }^{19}$.

\section{Statistical analysis}

All statistical analyses were performed using the PASW version 18 (SPSS Inc., Chicago, IL, USA). The greatest distance covered in 6 minutes by each healthy subject was identified from among the two 6MWT trials performed and then used in the following analyses. A descriptive analysis was made to summarize the population's demographic and clinical parameters. Student's t-tests were used to compare female and male subjects. Pearson's univariate correlation coefficients (r) were computed to examine the association between subject characteristics and 6MWD. A stepwise linear regression model was used to determine if any of the above variables were independent predictors of 6MWD. The mean 6MWD measured of the subjects in our study was compared to the mean predicted 6MWD derived from the eight previously published equations $^{8-14,20}$ according to each same age ranges. A p-value of $<0.05$ was considered significant for these analyses.

\section{Results}

1. Demographic, anthropometric, spirometric, and 6MWT data

Of the 278 subjects recruited for participation in this study, 19 were excluded because of smoking history of over 10 pack- 
Table 1. Characteristics of the study subjects

\begin{tabular}{|c|c|c|c|c|}
\hline Characteristic & Total $(n=259)$ & Male $(n=95)$ & Female $(n=164)$ & p-value* \\
\hline Age, yr & $36.6 \pm 10.83$ & $34.8 \pm 9.58$ & $37.7 \pm 11.38$ & 0.04 \\
\hline Height, $\mathrm{cm}$ & $164.5 \pm 8.65$ & $173.6 \pm 5.21$ & $159.2 \pm 5.18$ & $<0.001$ \\
\hline Weight, kg & $61.0 \pm 11.72$ & $73.2 \pm 8.21$ & $54.0 \pm 6.44$ & $<0.001$ \\
\hline $\mathrm{BMI}, \mathrm{kg} / \mathrm{m}^{2}$ & $22.4 \pm 2.85$ & $24.3 \pm 2.40$ & $21.3 \pm 2.50$ & $<0.001$ \\
\hline FVC, L & $3.6 \pm 0.88$ & $4.5 \pm 0.64$ & $3.0 \pm 0.42$ & $<0.001$ \\
\hline FVC, \%pred & $89.1 \pm 10.42$ & $90.2 \pm 10.07$ & $88.5 \pm 10.60$ & 0.186 \\
\hline $\mathrm{FEV}_{1}, \mathrm{~L}$ & $3.0 \pm 0.69$ & $3.7 \pm 0.56$ & $2.6 \pm 0.40$ & $<0.001$ \\
\hline $\mathrm{FEV}_{1}, \%$ pred & $96.2 \pm 11.11$ & $94.1 \pm 10.76$ & $97.4 \pm 11.18$ & 0.024 \\
\hline
\end{tabular}

Values are expressed as mean \pm SD.

${ }^{*}$ p-value between male and female.

BMI: body mass index; FVC: forced vital capacity; $\mathrm{FEV}_{1}$ : forced expiratory volume in one second.

Table 2.6MWT measures of the study subjects

\begin{tabular}{|c|c|c|c|c|}
\hline Characteristic & Total $(n=259)$ & Male (n=95) & Female $(n=164)$ & p-value* \\
\hline Resting HR, bpm & $78.6 \pm 9.35$ & $77.1 \pm 9.90$ & $79.5 \pm 8.93$ & 0.049 \\
\hline Resting $\mathrm{SpO}_{2}, \%$ & $97.9 \pm 0.85$ & $97.6 \pm 0.90$ & $98.2 \pm 0.74$ & $<0.001$ \\
\hline 6MWD, m & $598.5 \pm 57.92$ & $628.9 \pm 59.51$ & $580.9 \pm 47.80$ & $<0.001$ \\
\hline Borg after 6MWT & $0.19 \pm 0.334$ & $0.3 \pm 0.39$ & $0.15 \pm 0.287$ & 0.004 \\
\hline HR after 6MWT, bpm & $115.4 \pm 19.49$ & $112.5 \pm 21.63$ & $117.2 \pm 17.98$ & 0.064 \\
\hline$\%$ mHR after 6MWT & $63.0 \pm 10.48$ & $60.7 \pm 10.96$ & $64.4 \pm 9.97$ & 0.006 \\
\hline $\mathrm{SpO}_{2}$ after $6 \mathrm{MWT}, \%$ & $97.2 \pm 1.21$ & $96.7 \pm 1.35$ & $97.6 \pm 0.97$ & $<0.001$ \\
\hline Change in $\mathrm{SpO}_{2}, \%$ & $0.7 \pm 1.22$ & $1.0 \pm 1.50$ & $0.6 \pm 1.01$ & 0.025 \\
\hline
\end{tabular}

Values are expressed as mean \pm SD.

*p-value between male and female.

6MWT (D): 6 minute walking test (distance); HR: heart rate; $\mathrm{SpO}_{2}$ : oxygen saturation; \% mHR: percentage of the predicted maximum heart rate.

years $(\mathrm{n}=13)$ and high blood pressure $(\mathrm{n}=6)$. Characteristics of the remaining 259 healthy subjects are summarized in Table 1. The study population was composed of 95 males and 164 females, with a mean age of $36.6 \pm 10.83$ years, height $164.5 \pm 8.65$ $\mathrm{cm}$, weight $61.0 \pm 11.72 \mathrm{~kg}$, BMI $22.4 \pm 2.85 \mathrm{~kg} / \mathrm{m}^{2}$, and pre-bronchodilator $\mathrm{FEV}_{1} 96.2 \pm 11.11 \%$ predicted. Significant difference was observed in age, height, weight, BMI, FVC (L), $\mathrm{FEV}_{1}(\mathrm{~L})$, and $\mathrm{FEV}_{1}(\%$ pred) between male and female subjects.

The 6MWT data are shown in Table 2. The mean 6MWD was $598.5 \pm 57.92 \mathrm{~m}$ (range, $447-761 \mathrm{~m}$ ) for the group total and was $628.9 \pm 59.51 \mathrm{~m}$ (range, $447-761 \mathrm{~m}$ ) for male and $580.9 \pm 47.80 \mathrm{~m}$ (range, 454-720 m) for female, with significantly longer distances walked by males than females $(\mathrm{p}<0.001)$. Male and female subjects reached $60.7 \%$ and $64.4 \%$ of their maximum predicted heart rates, respectively, at the end of the test. The mean 6MWD of second walk was significantly longer than that of first walk $(595.0 \pm 58.27 \mathrm{~m}$ vs. $578.3 \pm 55.03$ $\mathrm{m}, \mathrm{p}<0.001$ ), suggesting a learning effect. We observed a small but significant difference between two groups with regards to resting heart rate, resting oxygen saturation, Borg values, and oxygen saturation measured at the end of the test. No clinically significant oxygen desaturation during 6MWT was observed.

\section{Univariate and multiple regression analysis}

Univariate correlation coefficients between the 6MWD and subject variables are presented in Table 3. Significant correlations with age, height, weight, and BMI were observed in the group total (Figure 1). However, gender differences were observed. While age and height were significantly correlated with 6MWD in male subjects, there was no significantly correlated variable in female subjects. In the stepwise multiple regression analysis using age, height, weight, and sex in the group total, height was identified as a single independent contributing factor to 6MWD $(\mathrm{p}<0.001)$, and the model explained $20.5 \%$ of the variability in 6MWD. The regression equation for esti- 
Table 3. Univariate correlation coefficients for 6MWD

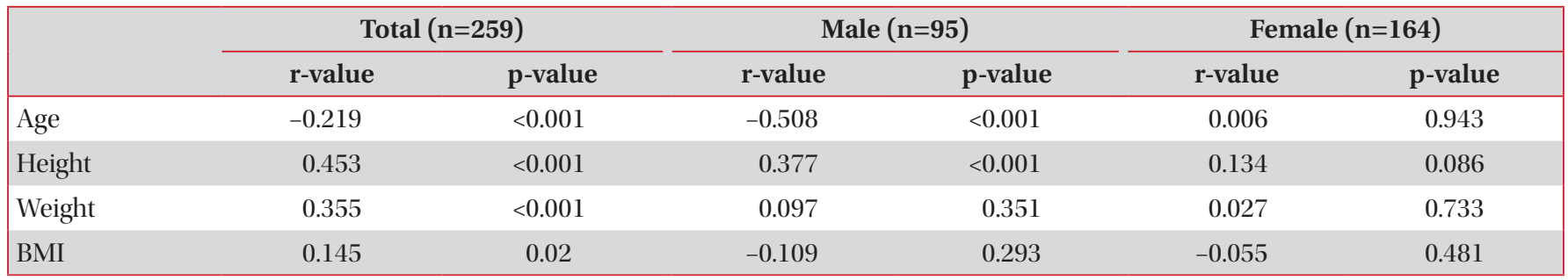

6MWD: six-minute walking distance; r-value: Pearson's correlation coefficient; BMI: body mass index.
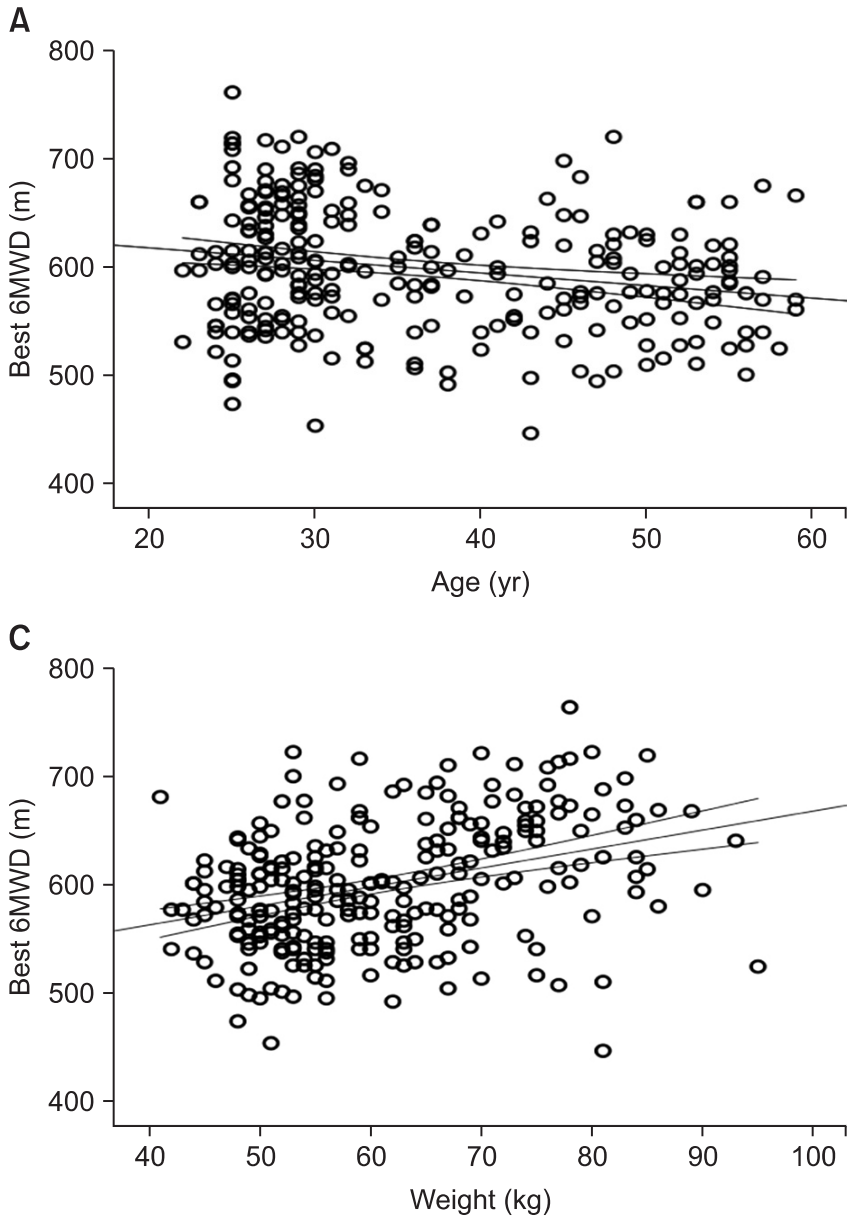

mating 6MWD is as follows: $6 \mathrm{MWD}(\mathrm{m})=105.7+2.99 \times$ height $(\mathrm{cm})\left(\mathrm{r}^{2}=0.205\right)$. In the group of 20-29 years, $\mathrm{sex}(\mathrm{p}=0.005)$ and height $(\mathrm{p}=0.028)$ were significant independent predictors and together explained $42.4 \%$ of the variability in 6MWD. In the group of 30-39 years, height $(\mathrm{p}<0.001)$ was an independent predictor, and this model accounted for $23.9 \%$ of the total variance for the 6MWD. According to the gender, age $(\mathrm{p}<0.001)$ and height $(\mathrm{p}=0.014)$ were significant independent predictors in the group of male and together explained $30.5 \%$ of the variability in 6MWD. No significant independent demographic or anthropometric factors predicting the $6 \mathrm{MWD}$ in the group of

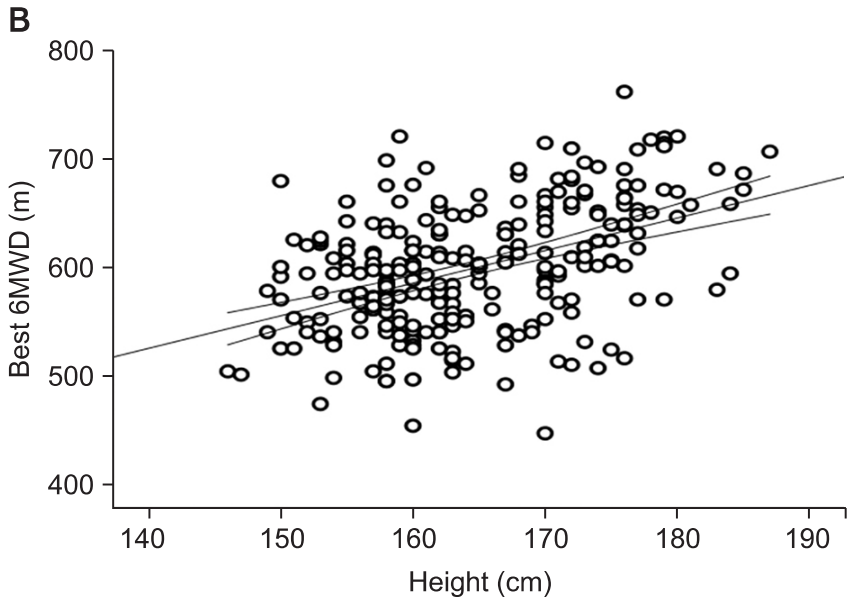

Figure 1. Relationships between six-minute walking distance (6MWD) and age $(\mathrm{r}=-0.219, \mathrm{p}<0.001)(\mathrm{A}), 6 \mathrm{MWD}$ and height $(\mathrm{r}=0.453, \mathrm{p}<0.001)(\mathrm{B})$, and $6 \mathrm{MWD}$ and weight $(\mathrm{r}=0.355, \mathrm{p}<0.001)$ (C). The linear regression line is superimposed, surrounded with the $95 \%$ confidence interval lines for the regression line.

female and of 40-59 years were observed (Table 4).

\section{Comparison with published regression equations}

Comparisons between the 6MWD measured in our subjects and predicted 6MWD, with a match for the same age range, from the reference equations derived in Caucasian $^{8-12}$, African $^{20}$ and Asian ${ }^{13,14}$ populations are shown in Table 5. Walk distance for our cohort was overestimated by four out of six equations derived from Caucasian and African populations, including Troosters et al. ${ }^{9}$, who overestimated the 
Table 4. Predicting model for $6 \mathbf{M W D}^{*}$

\begin{tabular}{|c|c|c|c|c|c|}
\hline \multicolumn{2}{|c|}{ Group } & Coefficient & Standard error & Standardized coefficient & p-value \\
\hline \multirow[t]{2}{*}{ Total } & Constant & 105.7 & 60.6 & & 0.082 \\
\hline & Height & 2.99 & 0.37 & 0.45 & $<0.001$ \\
\hline \multirow[t]{3}{*}{$20-29 \mathrm{yr}$} & Constant & 288.6 & 167.2 & & 0.087 \\
\hline & Sex & -47.0 & 16.39 & -0.383 & 0.005 \\
\hline & Height & 2.13 & 0.95 & 0.298 & 0.028 \\
\hline \multirow[t]{2}{*}{$30-39 \mathrm{yr}$} & Constant & 29.2 & 132.84 & & 0.827 \\
\hline & Height & 3.41 & 0.79 & 0.488 & $<0.001$ \\
\hline \multirow[t]{3}{*}{ Male } & Constant & 265.3 & 190.86 & & 0.168 \\
\hline & Age & -2.67 & 0.57 & -0.43 & $<0.001$ \\
\hline & Height & 2.63 & 1.06 & 0.23 & 0.014 \\
\hline
\end{tabular}

${ }^{*}$ Reference equations of six-minute walking distance (6MWD) are as follows.

Total: $6 \mathrm{MWD}(\mathrm{m})=105.7+2.99 \times$ height $(\mathrm{cm}) ; \mathrm{r}^{2}=0.205$.

$20-29$ yr: $6 \mathrm{MWD}(\mathrm{m})=288.6-47.0 \times \operatorname{sex}($ male $=0$, female $=1)+2.13 \times$ height $(\mathrm{cm}) ; \mathrm{r}^{2}=0.424$.

30-39 yr: 6 MWD $(\mathrm{m})=29.2+3.41 \times$ height $(\mathrm{cm}) ; \mathrm{r}^{2}=0.239$.

Male: $6 \mathrm{MWD}(\mathrm{m})=265.3-2.67 \times$ age $(\mathrm{yr})+2.63 \times$ height $(\mathrm{cm}) ; \mathrm{r}^{2}=0.305$.

There were no significant demographic or anthropometric variables predicting the 6MWD in the group of female and of 40-59 years.

Table 5. Measured and predicted 6MWD, for the same age range, from the equations derived in previous studies

\begin{tabular}{|c|c|c|c|}
\hline Study & Measured (m) & Predicted (m) & Measured-Predicted (m) \\
\hline Enright and Sherrill $^{8}$ & $582.5 \pm 49.89$ & $591.5 \pm 37.11$ & $-9.0 \pm 59.05$ \\
\hline Troosters et al. $^{9}$ & $580.5 \pm 44.30$ & $665.0 \pm 54.42$ & $-84.5 \pm 68.08^{*}$ \\
\hline Gibbons et al. ${ }^{11}$ & $598.5 \pm 57.92$ & $711.9 \pm 51.44$ & $-113.4 \pm 58.60^{*}$ \\
\hline Camarri et al. $^{10}$ & $581.4 \pm 48.67$ & $682.6 \pm 44.04$ & $-101.2 \pm 54.41^{*}$ \\
\hline Chetta et al. ${ }^{12}$ & $601.9 \pm 58.99$ & $608.2 \pm 39.17$ & $-6.3 \pm 54.06$ \\
\hline Ben Saad et al. ${ }^{20}$ & $582.5 \pm 49.89$ & $652.9 \pm 81.62$ & $-70.4 \pm 92.63^{*}$ \\
\hline Poh et al. ${ }^{14}$ & $585.2 \pm 48.90$ & $561.2 \pm 67.63$ & $24.0 \pm 51.97^{*}$ \\
\hline Alameri et al. ${ }^{13}$ & $602.8 \pm 59.09$ & $462.2 \pm 23.03$ & $140.6 \pm 52.40^{*}$ \\
\hline Present study & $598.5 \pm 57.92$ & $598.5 \pm 25.91$ & $0.1 \pm 50.98$ \\
\hline
\end{tabular}

* $\mathrm{p}<0.05$ by student t-test.

6MWD: six-minute walking distance.

distance walked by our subjects by $84.5 \pm 68.08 \mathrm{~m}(\mathrm{p}<0.001)$, Camarri et al. ${ }^{10}$ by $101.2 \pm 54.41 \mathrm{~m}(\mathrm{p}<0.001)$, Gibbons et al. ${ }^{11}$ by $113.4 \pm 58.60 \mathrm{~m}(\mathrm{p}<0.001)$ and Ben Saad et al. ${ }^{20}$ by $70.4 \pm 92.63$ $\mathrm{m}(\mathrm{p}<0.001)$. However, walk distance was underestimated by two equations derived in Asian populations, Poh et al. ${ }^{14}$ by $24.0 \pm 51.96 \mathrm{~m}(\mathrm{p}<0.001)$ and Alameri et al. ${ }^{13}$ by $140.6 \pm 52.40 \mathrm{~m}$ $(\mathrm{p}<0.001)$.

\section{Discussion}

To our knowledge, this is the first study to predict 6MWD in healthy Korean populations. The average 6MWD in these Korean populations was $598.5 \pm 57.92 \mathrm{~m}$. The regression equa- tion revealed that height was the most significant predictor of distance, explaining $20.5 \%$ of the distance variance. The 6MWD in these populations showed a considerable variability, ranging $447-761 \mathrm{~m}$, in line with previous studies ${ }^{9,10,12,14,20}$. However, the average 6MWD measured here was relatively shorter than those of Caucasian ${ }^{8-11}$ and North African studies ${ }^{20}$ and longer than those of Asian studies ${ }^{13,14}$. It is possible that this discrepancy reflects differences in how 6MWT protocol was conducted and differences in racial, demographic, and anthropometric factors of subjects recruited in each study. In addition, subjects' submaximal effort was also considered as a possibility. In this study, a 6MWT was performed twice and the better of the two tests was used for further analysis. In two studies ${ }^{9,20}$ consistent with our protocol, despite the fact that re- 
cruited subjects were relatively older than ours, their average $6 \mathrm{MWD}$ were longer than that of present study. Our subjects reached an average of $63 \pm 10 \%$ of their maximum predicted heart rates, while subjects in these studies reached an average of $77 \pm 15 \%, 79 \pm 13 \%$ of their mHRs, respectively. However, in the middle Asian study by Alameri et al. ${ }^{13}$, using single 6MWT protocol, the overall mean walking distance was $410 \pm 52 \mathrm{~m}$, which was significantly shorter than those in our study. Despite possessing similar age ranges as those of our subjects, the male and female subjects in Alameri et al's study ${ }^{13}$ reached an average of $44 \%$ and $47 \%$ of their mHRs, respectively. This is natural in view of that there is a high prevalence of physical inactivity among Saudis ${ }^{21}$. In addition to a subject's level of daily physical activity, attitude or psychological factors may also influence the degree of submaximal effort ${ }^{22}$.

Several demographic and anthropometric factors can influence the 6MWT performance in healthy subjects, showing $34-78 \%$ of the variance in the $6 \mathrm{MWD}^{8-12,14,20}$. Among them, age, height, weight, and sex were the most common variables used to predict 6MWD. However, in our model, height was the only reliable predictor of distance, explaining $20.5 \%$ of the distance variance. This low variability is similar to that of recent study by Alameri et al. ${ }^{13}$; wherein twenty five percent of the variability of 6MWD was explained by age and height. Not surprisingly, a taller height is associated with a longer stride, which generates a longer distance ${ }^{8,9,14}$. In the univariate analysis of our series, age and height were significantly correlated with 6MWD in male subjects, but no significant variables were observed in female subjects. In the end, sex and age could not enter final model in the group total. However, sex was significant independent predictor in the younger age group. In the group of female and of 40-59 years, no significant independent demographic or anthropometric factors predicting the 6MWD were observed. As in the case of previous studies $^{11,12}$, the different level of peripheral muscle conditioning or physical fitness of the participants in this study, particularly in female subjects, may be partly responsible for this, because most of the female subjects in their forties and fifties were hospital sanitation workers who were more physically active than younger subjects.

In present study, while the reference equations derived in Caucasian $^{8-12}$ and North African ${ }^{20}$ populations tend to overestimate the distance walked by Korean subjects, two Asian equations $\mathrm{s}^{13,14}$ underestimate it. The 6MWT has been widely used in clinical settings as an outcome assessment with regards to therapeutic or prognostic determinants. Clearly, these previously reported equations can provide misleading information when applied to Korean patients. It is therefore necessary to develop reference equations specific to Koreans.

This study was limited by several factors. First, our subjects consisted of middle-aged subjects. Therefore, the reference equation in the present study may predict 6MWD in Korean patients with cardio-respiratory dysfunction at a relatively young age. However, since most chronic obstructive pulmonary disease patients are older than the present cohort, there may be some controversy to apply this reference equation to wider populations. Second, the sample studied in present study included medical personnel, workers, medical students, and visitors at a local referral hospital and may not be representative of the entire Korean population. A well-organized nationwide study is needed to solve this problem. Third, the study did not attempt to evaluate the degree to which typical daily physical activity and psychological factors affected 6MWD.

In conclusion, the average 6MWD in these Korean populations was $600 \mathrm{~m}$. The regression equation revealed that height was the most significant predictor of distance, explaining $20.5 \%$ of the distance variance.

\section{Conflicts of Interest}

No potential conflict of interest relevant to this article was reported.

\section{References}

1. Pauwels RA, Buist AS, Calverley PM, Jenkins CR, Hurd SS; GOLD Scientific Committee. Global strategy for the diagnosis, management, and prevention of chronic obstructive pulmonary disease. NHLBI/WHO Global Initiative for Chronic Obstructive Lung Disease (GOLD) Workshop summary. Am J Respir Crit Care Med 2001;163:1256-76.

2. Ries AL, Bauldoff GS, Carlin BW, Casaburi R, Emery CF, Mahler DA, et al. Pulmonary rehabilitation: Joint ACCP/ AACVPR evidence-based clinical practice guidelines. Chest 2007;131(5 Suppl):4S-42S.

3. Nici L, Donner C, Wouters E, Zuwallack R, Ambrosino N, Bourbeau J, et al. American Thoracic Society/European Respiratory Society statement on pulmonary rehabilitation. Am J Respir Crit Care Med 2006;173:1390-413.

4. Bruce RA. Methods of exercise testing. Step test, bicycle, treadmill, isometrics. Am J Cardiol 1974;33:715-20.

5. Cox NJ, Hendriks JC, Binkhorst RA, Folgering HT, van Herwaarden CL. Reproducibility of incremental maximal cycle ergometer tests in patients with mild to moderate obstructive lung diseases. Lung 1989;167:129-33.

6. Clinical exercise testing with reference to lung diseases: indications, standardization and interpretation strategies. ERS Task Force on Standardization of Clinical Exercise Testing. European Respiratory Society. Eur Respir J 1997;10:2662-89.

7. ATS Committee on Proficiency Standards for Clinical Pulmonary Function Laboratories. ATS statement: guidelines for the six-minute walk test. Am J Respir Crit Care Med 2002;166: 111-7. 
8. Enright PL, Sherrill DL. Reference equations for the sixminute walk in healthy adults. Am J Respir Crit Care Med 1998;158(5 Pt 1):1384-7.

9. Troosters T, Gosselink R, Decramer M. Six minute walking distance in healthy elderly subjects. Eur Respir J 1999;14:2704.

10. Camarri B, Eastwood PR, Cecins NM, Thompson PJ, Jenkins S. Six minute walk distance in healthy subjects aged 55-75 years. Respir Med 2006;100:658-65.

11. Gibbons WJ, Fruchter N, Sloan S, Levy RD. Reference values for a multiple repetition 6-minute walk test in healthy adults older than 20 years. J Cardiopulm Rehabil 2001;21:87-93.

12. Chetta A, Zanini A, Pisi G, Aiello M, Tzani P, Neri M, et al. Reference values for the 6-min walk test in healthy subjects 2050 years old. Respir Med 2006;100:1573-8.

13. Alameri H, Al-Majed S, Al-Howaikan A. Six-min walk test in a healthy adult Arab population. Respir Med 2009;103:1041-6.

14. Poh H, Eastwood PR, Cecins NM, Ho KT, Jenkins SC. Six-minute walk distance in healthy Singaporean adults cannot be predicted using reference equations derived from Caucasian populations. Respirology 2006;11:211-6.

15. Choe KH, Park YJ, Cho WK, Lim CM, Lee SD, Koh YS, et al.
The effect of pulmonary rehabilitation in patients with chronic lung disease. Tuberc Respir Dis 1996;43:736-45.

16. Kim C, Park YB, Mo EK, Choi EH, Nam HS, Lee SS, et al. Predicting oxygen uptake for men with moderate to severe chronic obstructive pulmonary disease. Tuberc Respir Dis 2008;64:433-8.

17. Kim YS, Byun MK, Jung WY, Jeong JH, Choi SB, Kang SM, et al. Validation of the Korean version of the St. George's respiratory questionnaire for patients with chronic respiratory disease. Tuberc Respir Dis 2006;61:121-8.

18. Lee JH, Shin HI, Jeong YS. Reliability of 6-minute walk test in stroke patients. J Korean Geriatr Soc 2005;9:39-44.

19. Standardization of spirometry, 1994 update. American Thoracic Society. Am J Respir Crit Care Med 1995;152:1107-36.

20. Ben Saad H, Prefaut C, Tabka Z, Mtir AH, Chemit M, Hassaoune $\mathrm{R}$, et al. 6-minute walk distance in healthy North Africans older than 40 years: influence of parity. Respir Med 2009;103:74-84.

21. Al-Hazzaa HM. Prevalence of physical inactivity in Saudi Arabia: a brief review. East Mediterr Health J 2004;10:663-70.

22. King B, Cotes JE. Relation of lung function and exercise capacity to mood and attitudes to health. Thorax 1989;44:402-9. 